This item was submitted to Loughborough's Research Repository by the author.

Items in Figshare are protected by copyright, with all rights reserved, unless otherwise indicated.

\title{
Observations of solder paste reflow by means of electrical measurements
}

PLEASE CITE THE PUBLISHED VERSION

PUBLISHER

(C) IEEE

LICENCE

CC BY-NC-ND 4.0

\section{REPOSITORY RECORD}

Mannan, Samjid H., David A. Hutt, David C. Whalley, and Paul P. Conway. 2019. "Observations of Solder Paste Reflow by Means of Electrical Measurements". figshare. https://hdl.handle.net/2134/3930. 
This item was submitted to Loughborough's Institutional Repository (https://dspace.lboro.ac.uk/) by the author and is made available under the following Creative Commons Licence conditions.

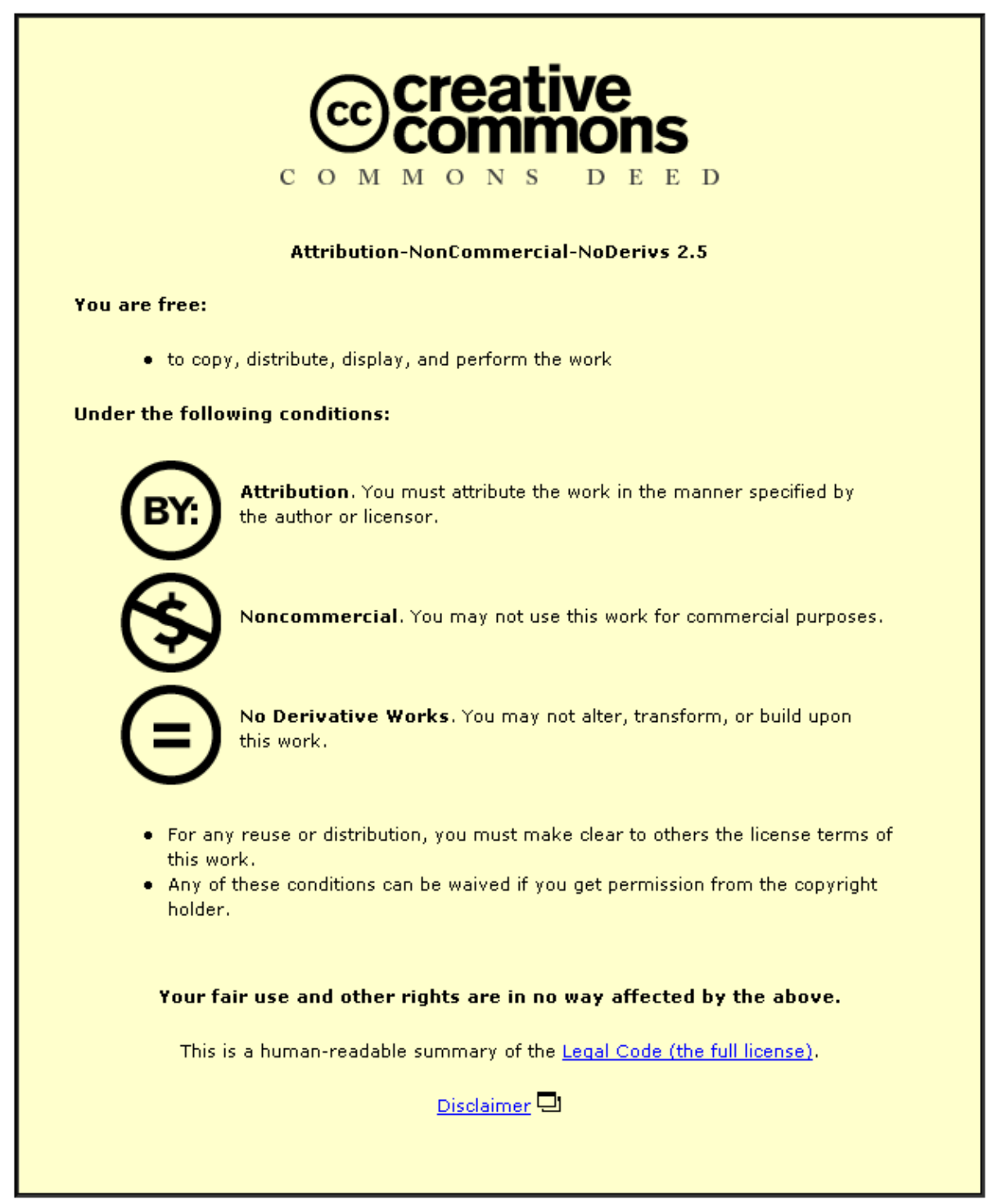

For the full text of this licence, please go to: http://creativecommons.org/licenses/by-nc-nd/2.5/ 


\title{
Observations of Solder Paste Reflow by means of Electrical Measurements
}

\author{
S H Mannan, \\ King's College London, \\ Dept. of Mech. Eng., Strand, London WC2R 2LS, UK \\ D A Hutt, D C Whalley, P P Conway \\ Loughborough University, \\ Dept. of Manf. Eng., Loughborough LE11 3TU, UK
}

\begin{abstract}
This paper presents a method for exploring the changes occurring at the surfaces of solder particles during the reflow soldering process. The method involves measuring changes in electrical resistance of a sample of paste as a function of test voltage. The results are used to estimate the size and nature of electrical contact spots between the particles, and how these depend on temperature and time. The activation energy of the process responsible for increasing the size of contact spots is deduced for RA and RMA type fluxes and it is shown that sintering is not the dominant mechanism for increasing contact size. These results, together with a programme of CFD studies are expected to help improve solder paste formulations.
\end{abstract}

\section{Introduction}

Electronics miniaturization is leading to smaller solder joints for electronic components. For example, Chip Scale Package (CSP) and flip chip solder joints are significantly smaller than those for traditional SMT components, and the balance of physical and chemical processes during reflow is altered, due for example to the change in surface area to volume ratio. These changes mean that solder paste performance may not be satisfactory for these small volume solder joints, necessitating the development of new solder paste formulations and modifications to the flux chemistry. There is a lack of understanding of the processes occurring during coalescence of the solder paste, principally due to the absence of suitable investigative tools to monitor this process.

The method presented here involves measuring the electrical resistance in a sample of paste at a fixed or zero frequency. The technique is similar to that used in Electrical Impedance Spectroscopy (EIS) experiments [1], where the frequency is swept through a range, typically $0.01 \mathrm{~Hz}$ to $\mathrm{MHz}$. However, EIS is not suitable for examining changes in a system experiencing rapid change. Another advantage of the proposed method reported here, is that the non-linear characteristics of the current-voltage curve can be used to help determine the changing nature of the contacts between the solder particles as the reflow process progresses. In particular, the presence or absence of thin semiconducting films across the contact spots can be detected.

This paper first outlines the changes in electrical resistance that occur in $\mathrm{Sn} / \mathrm{Pb}$ solder paste as the temperature is raised towards $\sim 110^{\circ} \mathrm{C}$, driving off the volatiles in the paste. It is shown that mere mechanical contact is insufficient to create percolating electrical pathways in the paste, and that mechanical abrasion, or the fluxing action of the vehicle, must allow penetration of the surface oxide layer before conduction can begin to develop. Once conduction has begun, resistance vs. time and resistance vs. temperature curves allow an estimation of the size of the electrical contact spots (the areas of contact between particles free of oxides and other contaminants), and the growth rate of these contact spots. It is demonstrated that the contact spots between particles are the same order of magnitude as the contact spots between particles and solderable terminations on the substrate, and that while the paste resistance can be homogenous, instances of heterogeneous conduction also occur. Furthermore, the monotonic decrease in resistance measured in the paste, observed even over a period of several days, shows that reoxidation of the contacting solder surfaces is not a significant factor, at least in the bulk of the paste as opposed to the particles at the sample surface.

Metal powders in contact near the metal melting temperature are expected to undergo sintering, but the rate of growth of the oxide spots suggests that sintering is not the dominant mechanism for increasing contact spot size. It is expected that reactions between the oxide and flux are mainly responsible, though creep of the solder may also play a part. The values of activation energies deduced from the experiments are consistent with chemical action and creep.

\section{Experimental Technique}

The apparatus for the electrical measurements is sketched in fig. 1. It consists of a hot plate for heating the paste, a signal generator for applying the desired test voltage signal and resistance measurement equipment.

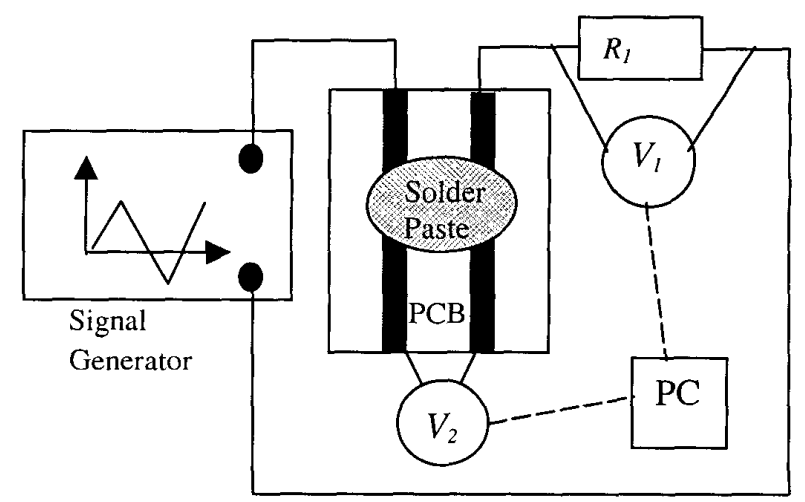

Figure 1. Experimental setup for measuring solder paste resistance 
Solder Paste is placed between the $\mathrm{Cu}$ traces on a Printed Circuit Board (PCB) which is in turn placed on a hotplate. A thermocouple is partially embedded in the solder paste (not shown in fig.1) to allow precise control of temperature. As the paste is heated, the voltages $V_{1}$ and $V_{2}$ are recorded to allow measurement of the paste resistance, $R$, through the formula:

$$
R=R_{1} V_{2} / V_{1}
$$

Measurements were taken at constant temperature as well as during ramped temperature profiles typical of SMT reflow ovens. All experiments were conducted either in air or in partial vacuum. The cross sectional area of the solder paste deposit was typically $1 \mathrm{~mm}^{2}$, and the distance between tracks varied from $0.5-10 \mathrm{~mm}$.

Typical peak test voltages across the solder sample were of the order of $I^{\prime} \mathrm{V}$ during the early stages of the experiments when the solder resistance was high (M $\Omega$ or higher), but dropped to $\sim 100 \mathrm{mV}$ as the resistance in the paste dropped to the $10 \mathrm{~m} \Omega$ mark as a result of the limited current drive capability of the signal source. The experimental setup could be improved by having a feedback amplifier boost the signal across the paste so that it could be kept at a constant level throughout the experiment. A selection of experiments, detailed in Section 3, were carried out at much lower voltages $(<10 \mathrm{mV})$ using a mOhmmeter on a dry circuit test setting to measure resistance.

\section{Electrical Contact Spots Between Solder Paste Particles}

Solder paste at room temperature typically exhibits an extremely low DC conductance. What current does flow is mainly due to ionic migration between the electrodes. However if the paste is subjected to prolonged shear between plane surfaces, the oxide layers on the particles can be partially removed by mechanical abrasion, and the resistivity drops to $\sim 1 \Omega \mathrm{m}$. [1] By contrast heating the paste to just below the reflow temperature results in resistivities of $\sim 10^{-6} \Omega \mathrm{m}$, which is comparable to bulk $\mathrm{SnPb}$ solder resisitivity of $\sim 10^{-7} \Omega \mathrm{m}$.

The resistance of solder paste (and also dry solder powder) can also be reduced by subjecting the paste to high electric fields $\left(\sim 10^{4} \mathrm{~V} / \mathrm{m}\right)$, causing dielectric breakdown in the insulating (oxide) layers surrounding the solder particles. In this case, the high current densities through the particles which are in electrical contact melt the particles and cause continuous solder bridges to form across the electrodes. This process is not of further interest for the purposes of this paper.

The average gap between solder particles in solder paste is expected to be $\sim 1 \mu \mathrm{m}$. [2] However, the high volume fraction of particles would be expected to cause percolation in the absence of insulating layers (e.g. metal oxides or rosin from the flux), or any physical mechanism (e.g. electrostatic repulsion) keeping the particles apart. In order to confirm that simple mechanical contact between particles is insufficient to break through the insulating layers in the absence of heat, a partial vacuum was applied to a paste sample at room temperature, allowing the volatiles in the paste to be driven off, and forcing the particles into mechanical contact by increasing the volume fraction of particles. The results showed that paste would still not conduct a DC current unless external pressure $\left(-10^{5} \mathrm{~Pa}\right.$, caused by the rubber seal on the vacuum equipment pressing against the paste) is applied, in which case resistivities of $10^{-1}-10^{-2} \Omega \mathrm{m}$ were observed.

The next set of experiments involved measurement of resistance in samples of varying lengths, in order to test whether paste conduction was homogeneous or dominated by a few particle contacts only (causing heterogeneous conduction). If paste conduction is relatively homogeneous (little variation in particle-particle contact resistance), then it is easy to extract an average particle-particle resistance and make deductions on the nature of the contact. The simplified geometry associated with a homogeneous conductivity system is sketched in Figure 2.

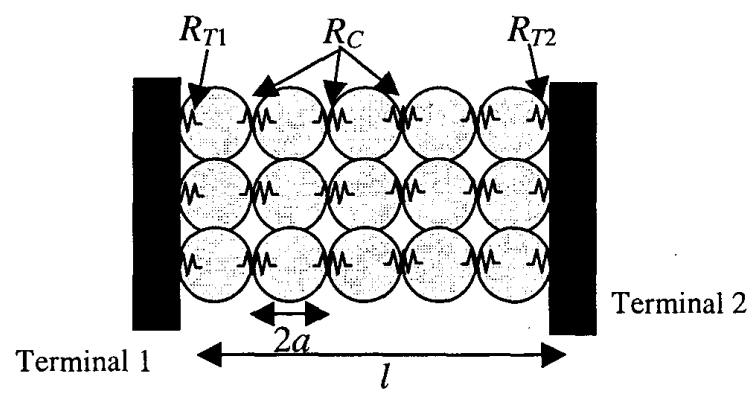

Figure 2. Chains of solder particles enabling conduction

The solder paste resistance is dominated by contact spots between particles where the insulating layers are thin enough to allow conduction or have been completely disrupted. These resistances have been labelled $R_{C}$, between particles, and $R_{T i}$ between a particle and a terminal. We see that the terminals are connected by a number of chains of solder spheres, each with $l /(2 a)$ particles. If the cross-sectional area of solder is $A$ then we have approximately $A / 4 a^{2}$ chains in parallel, each with a resistance of $R_{c} l /(2 a)$. Similarly the resistance associated with the terminals, with a contact area, $A_{T}$, with the paste, is $\left(R_{T 1}+R_{T 2}\right) 4 a^{2} / A_{T}$, giving a total resistance, $R$ of

$$
R=4 a^{2}\left(R_{T 1}+R_{T 2}\right) / A_{T}+2 a R_{c} l / A
$$

We thus see that the measuring $R$ against $l$ should give a straight line. However in practise, we cannot extend $l$ without disturbing the paste, or introducing a new terminal contact resistance. Hence we need to measure resistance between pairs of terminals; $i, j$, and eliminate the unknown terminal contact resistances. If the resistance between pairs of terminals is denoted $R_{i j}$, the length of solder paste deposit denoted by $l_{i j}$, and the contact resistance associated with each terminal $R_{i}$, then we obtain

$$
R_{i j}=R_{i}+R_{j}+\alpha l_{i j}
$$

Where $\alpha$ is given by $2 a R_{c} / A$ and $R_{i}$ by $4 a^{2} R_{T_{i}} / A_{T}$. If we measure the resistances between 4 terminals $(i, j=1 \ldots 4)$, we obtain 6 measurements, and have 5 unknowns $\left(R_{i}, \alpha\right)$. Using 
the first 5 measurements to solve for the unknowns allows prediction of the result of the 6th measurement.

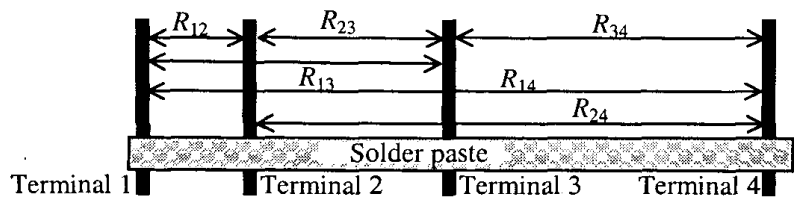

Figure 3. Simultaneous Measurement of the $R_{i j}$

Using this technique, good agreement between the model and experiment is obtained for a number of cases, while a number of instances require unphysical values for the $R_{i}$ or $\alpha$, or fail to correctly predict the $6^{\text {th }}$ measurement, indicating that the model does not apply. This is presumably due to the current being channelled through a small number of the available paths due to large variations in inter-particle contact resistance. Table 1 gives some representative values of $\alpha$ and the $R_{i}$ together with predicted and measured values of the $6^{\text {th }}$ measurement. Column one lists the maximum temperature reached by the paste, and the length of time for which this temperature was maintained.

Table 1. Fitted and measured parameter values from eq. 3

\begin{tabular}{|l|l|l|l|l|}
\hline $\begin{array}{l}\text { Temp. }{ }^{\circ} \mathrm{C} \\
\text { @Time } \\
\text { minutes }\end{array}$ & $\begin{array}{l}\alpha \\
\Omega / \mathrm{mm}\end{array}$ & $\begin{array}{l}\text { Calculated Values } \\
\text { for the } R_{i}\end{array}$ & $\begin{array}{l}\text { Predicted } \\
6^{\text {th }} R_{i j}\end{array}$ & $\begin{array}{l}\text { Measured } \\
6^{\text {th }} R_{i j}\end{array}$ \\
\hline $168 @ 2$ & 0.01 & $-55.27 .21 .2 \mathrm{~m} \Omega$ & $46.8 \mathrm{~m} \Omega$ & $46.8 \mathrm{~m} \Omega$ \\
\hline $130 @ 3$ & 0.06 & $10132284 \mathrm{~m} \Omega$ & $138 \mathrm{~m} \Omega$ & $135 \mathrm{~m} \Omega$ \\
\hline $96 @ 2$ & 0.5 & $1.31 .72 .06 .5 \Omega$ & $3.8 \Omega$ & $3.3 \Omega$ \\
\hline $90 @ 5$ & 173 & $-7013.5109 \Omega$ & $803 \Omega$ & $805 \Omega$ \\
\hline
\end{tabular}

Negative values of the $R_{i}$ in table 1 are unphysical but can be attributed to measurement errors and non-uniform cross sectional areas of the paste when the proportion of total resistance due to the $\alpha$ term in eq.(3) dominates. However there are also numerous instances, particularly when the resistance is still high, of negative values of $\alpha$ together with inaccurate predictions of the $6^{\text {th }}$ resistance measurement. In these cases the homogeneous conduction models are not applicable and it is not possible to estimate contact spot size.

Typical resistance values for large resistances (when the paste has not been heated above $\sim 110^{\circ} \mathrm{C}$ ) give $R_{i} \sim 1-10 \Omega$, while $\alpha \sim 1-100 \Omega / \mathrm{mm}$. Similarly when the paste has been heated above $\sim 110^{\circ} \mathrm{C}$, we see $R_{i} \sim 10 \mathrm{~m} \Omega, \alpha \sim 0.01 \Omega / \mathrm{mm}$. In both cases the $l_{i j}$ are $-1 \mathrm{~mm}, A \sim 1 \mathrm{~mm}^{2}$ and $A_{T} \sim .01 \mathrm{~mm}^{2}$.

Given that the solder particle diameter is $\sim 10 \mu \mathrm{m}$, the individual contact resistance between particles, $R_{c}$ can be estimated from equations (2) and (3); it is $\sim 50 \Omega-5 \mathrm{k} \Omega$ below $\sim 110^{\circ} \mathrm{C}$, and $\sim 0.5 \Omega$ above $-110^{\circ} \mathrm{C}$. The terminal resistances, $R_{T i}$, are also of these magnitudes, irrespective of whether the finish on the terminals is gold or solder. Holm's theory of electrical contacts [3] can be used to estimate the contact area between particles, assuming that a thin film is not present at the contact spots. For a single contact spot between particles of radius $r$, theory predicts the contact resistance $R_{c}$ to be given by:-

$$
R_{c}=\rho / 2 r
$$

Where $\rho$ is the conductivity of the metal which for solder is $10^{-7} \Omega \mathrm{m}$. For a $50 \Omega$ resistance, we would therefore expect $r$ $\sim 3 \mathrm{~nm}$, while for $.5 \Omega$ resistance, $0.3 \mu \mathrm{m}$ is expected. Equation 4 predicts contact spot sizes below $0.03 \mathrm{~nm}$ for values of $\mathrm{Rc}$ greater than $5 \mathrm{k} \Omega$, so it is likely that eq.(2) is no longer applicable. At this stage however it is not possible to determine whether thin films are present between the particles at the contact spots, adding to the contact spot resistance. The voltage - current relationships at the contacts can help detect the presence of thin films, and this is discussed in section 5 .

\section{Contact Spot Variation with time}

As solder paste is heated, up to $\sim 110^{\circ} \mathrm{C}$, the volatiles are driven off, bringing the particles into close contact. Above $\sim 110^{\circ} \mathrm{C}$, chemical reactions between the flux and solder surface are speeded up, resulting in rapid contact spot growth. Sintering is also expected to be present, causing solder necks to grow between particles where they make contact. However, sintering growth models predict growth rates which are too small for it to be the dominant process. Sintering models predict the spot radius to grow as $r \sim t^{n}$, where $n \sim 0.5$ [4]. By contrast, the rates that are deduced from eq.(4) show $r \sim t^{n}$ where $1<n<2$ when solder is kept at constant temperature, below its melting point. Samples of paste which were heated at $170^{\circ} \mathrm{C}$ for several hours were examined using optical microscopes and SEM, but the presence of flux prevented direct observation of the necks between particles, even after boiling in solvent for several hours. Figure 4 shows an optical photograph of the paste, looking for inter-particle necks, and figure 5 shows an SEM micrograph. Figure 6 shows the same sample after micro-sectioning but the extreme distortion of the particles is thought to be a result of the cross-sectioning and polishing operations, and it is therefore impossible to detect sintering directly in this manner.

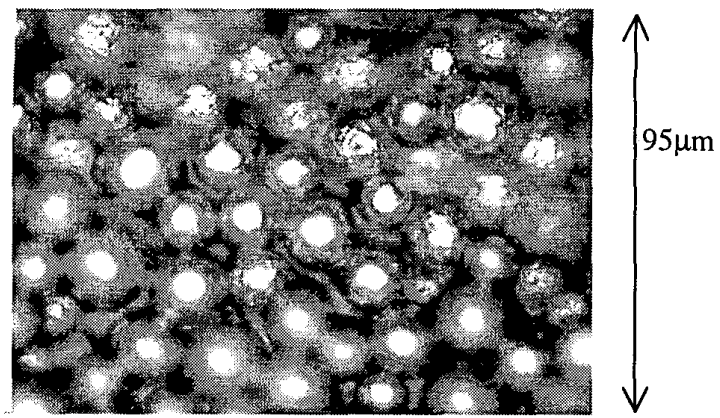

Figure 4. Lried solder paste 


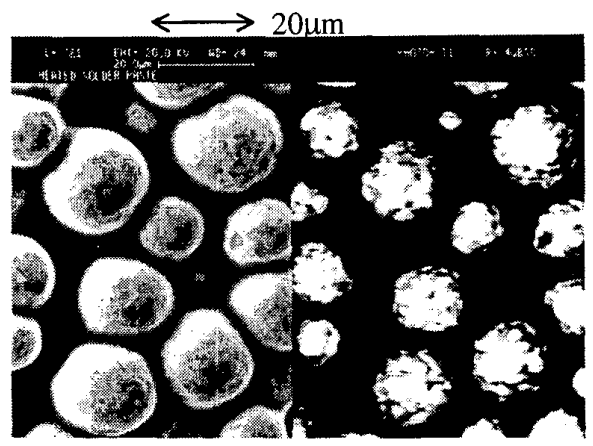

Figure 5. SEM showing $\mathrm{Sn}$ and $\mathrm{Pb}$ rich phases

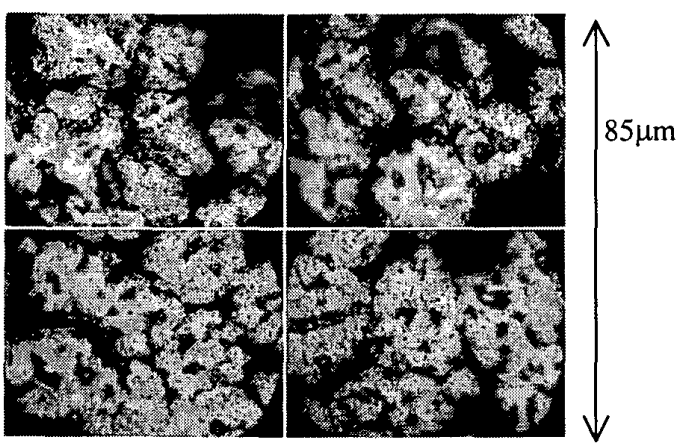

Figure 6. Dried paste cross section ( $3 \mathrm{~s}$ etch in nital)

Above the reflow temperature of the solder, the particles coalesce, expelling flux from the interior of the deposit. This expelled flux is thought to be important as it acts as a source of fresh flux to the outer layers of solder, but it also has a secondary effect of briefly $(\sim 1 \mathrm{~s})$ pushing the reflowing solder particles out of contact with the terminals. The solder rests on a cushion of flux before gravity acts to re-establish solder contact with the terminals. The results of one Resistance Vs temperature and time plot are shown in fig.7. Here we see the rapid decrease of resistance at $120^{\circ} \mathrm{C}$, and the further drop in resistance at $174^{\circ} \mathrm{C}$, which may be caused by local variations of paste temperature allowing paste to reflow in areas that are not in direct contact with the thermocouple. The further drop in paste resistance as the paste cools down to room temperature is attributable to the temperature dependence of resistance within bulk solder and the terminals.

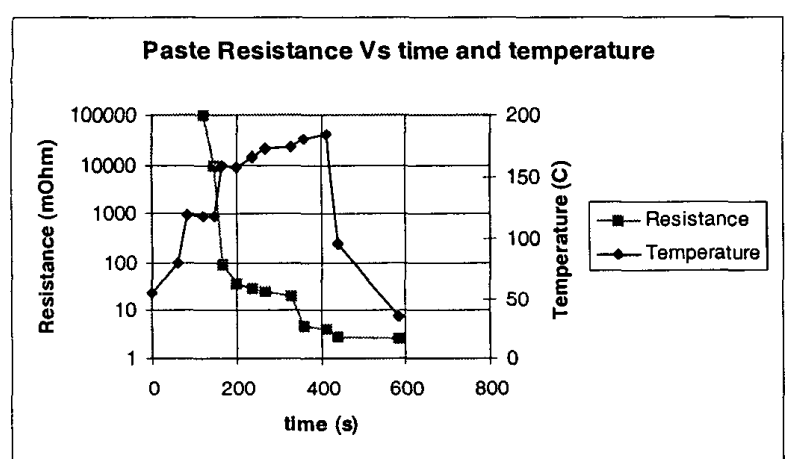

Figure 7. Paste heated to just below the reflow temperature
The variation of resistance with time at constant temperature is shown in fig.8, where a RA flux based paste is used. In this case the data can be fitted approximately to an $R$ $\sim 1 / t^{1.6}$ model but in general, it is found that $R \sim 1 / t^{1-2}$, or that a single $R \sim 1 / t^{\mathrm{n}}$ fit is not applicable over the entire duration of the experiment. Furthermore even in fig.8, the origin of the time axis was chosen to give the straightest line fits in the log$\log$ graph. The ambiguity in the time axis arises from the fact that the temperature in the paste cannot be instantaneously ramped up to the target temperature, so that $t$ lies somewhere between the time elapsed since the start of the experiment, and the time elapsed since the target temperature was attained. The Resistance axis is also adjusted to obtain the contact resistance by subtracting the bulk solder and terminal resistances ( $5.7 \mathrm{~m} \Omega$ in fig.8). Using fig. 8 we can attempt to calculate the activation energy $\left(E_{a}\right)$ of the process responsible for widening the contact spots.

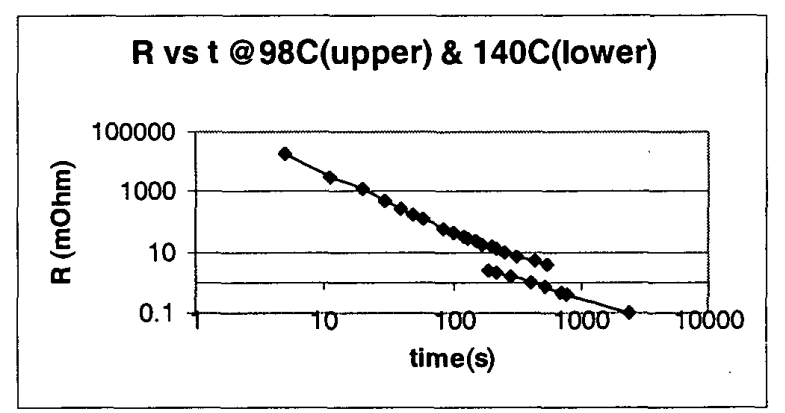

Figure 8. Contact resistance in a RA paste

If the data in fig. 8 is represented as $R=C l t^{n}$, where $C$ is a constant at a given temperature, then we can calculate the variation of $C$ with temperature. Assuming that the mechanism widening the contact spots obeys Arrhenius equation, then the variation of $R$ with $T$ and $t$ may be given as:-

$$
R=C^{\prime} \mathrm{e}^{-E a k T} t^{-n}
$$

Where $C^{\prime}$ is a constant depending on the geometry of the experiment and chemical kinetics, and $E_{a}$ is the activation energy. Figure 8 gives a value of $n$ of 1.6, and also the ratio of values for $C$ deduced from fig. 8 at $98^{\circ} \mathrm{C}$ and $140^{\circ} \mathrm{C}$. Equation 5 generates a value of $E_{a}=0.49 \mathrm{eV}$. This result is suggestive of solder creep $\left(E_{a}=0.48 \mathrm{eV}\right)$. Similarly, a value of $1 \mathrm{eV}$ is obtained for an RMA paste using eq.(5), and $n=1.9$. There are large errors in these values because of the difficulty in estimating $n$ from curves such as fig.8. Nevertheless, the reported approach has the advantage that it singles out the process responsible for widening the contact spots, ignoring the other paste reactions during heating which would have been picked up by conventional Differential Scanning Calorimetry (DSC). These values for $E_{a}$ can be compared to a value of $1.74 \mathrm{eV}$ reported for oxide removal on $\mathrm{Cu}$ surfaces by Sn without flux. [5]

\section{Variable Voltage experiments}


Electrical Impedance Spectroscopy is a method whereby the impedance response of a sample is measured over a range of different frequencies. In principle this is a very promising technique for examining changes in the sample e.g. solder paste, since the contributions of bulk and interfacial characteristics may be separated out. [6] The major limitations of the technique are that the sample must have changed little in the time taken to make the measurement (effectively cutting off the low frequency end of the spectrum) and that the effects of non-linear conduction are not included.

Non linear electrical conduction between solder spheres is expected either because of Ohmic heating at the contact spots or because of the presence of thin films (organic and oxides) between the particles through which a variety of mechanisms may contribute to conduction. The transport of electrons across a thin film may be barrier-limited (conduction is limited by the transfer of charge into the film) or bulk-limited (transport of charge across the film limits conduction). If barrier-limited, then Shottky emission or electron tunnelling determine conduction, while bulk-limited mechanisms include ionic or intrinsic conduction, Space-Charge limited conduction or Poole-Frenkel Emission. Each mechanism has a characteristic dependence on temperature and voltage, but it is not always possible to distinguish between them experimentally. [7]

Some preliminary measurements on non-linear conduction between particles have been made using a function generator for the voltage (see fig.1). In the latter stages of heating, when the resistance is relatively stable, it seems that Ohmic heating is occurring, as the resistance rises with applied voltage. This is illustrated in fig.9. These measurements were taken on solder paste that had been heated at $60-90^{\circ} \mathrm{C}$ for 15 minutes. The voltage source was set to generate a triangular waveform of peak voltage $\pm 1.5 \mathrm{~V}$, at $0.05 \mathrm{~Hz}$, until conduction occurred in the paste. Then the voltage was increased to its Open Circuit maximum of $10 \mathrm{~V}$ since only $\sim 100 \mathrm{mV}$ was generated across the sample as the resistance of the paste drops. The measurements in fig. 8 are shown 80 s after conduction begins.

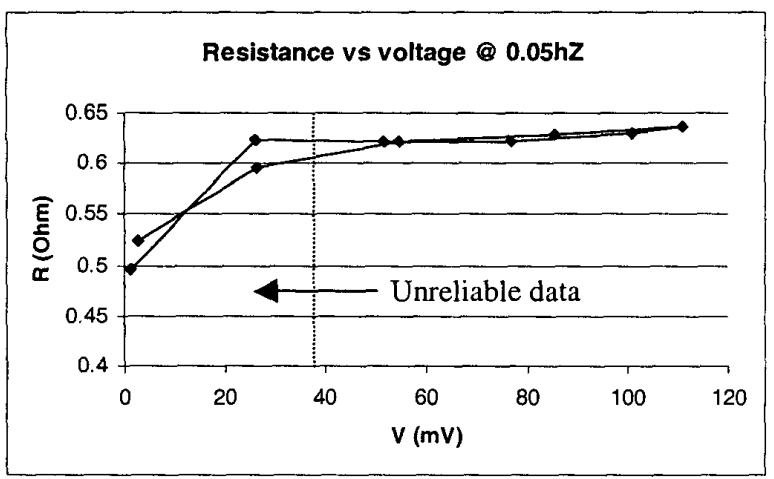

Figure 9. Evidence for Ohmic Heating (10s of data shown, representing one triangular pulse)

It should be noted that because the paste resistance is calculated by eq.(1), that when $V_{2}$ is small $(<30 \mathrm{mV})$ large errors can be made in calculating the resistance. These errors are probably responsible for the large variations in resistance seen at low voltages. Resistance vs. Voltage graphs recorded as the paste just begins to conduct are distorted by the fact that the resistance of the paste drops significantly during the course of one cycle. Figure 10 shows the $R-V$ characteristic just after conduction begins, together with a corrected version which factors out the overall drop in resistance with time. The latter shows more clearly the resistance rise with voltage, and also a resistance drop which may indicate film breakdown or softening of the metal due to heat - however, an unambiguous interpretation of the data at this stage is not possible.

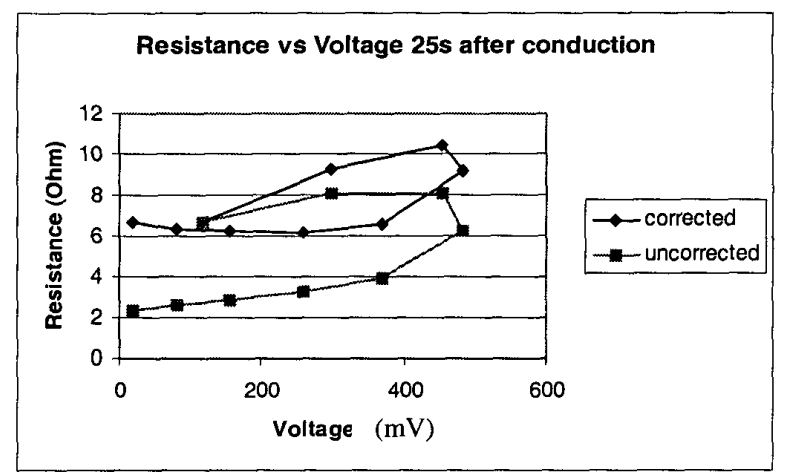

Figure 10. Resistance-voltage graph

Improvements to the measurements could occur by boosting the voltage across the paste by the use of an amplifier circuit, or by increasing the applied frequency to minimise the effect of the resistance drop during the course of the measurement. The latter approach was taken in fig. 11 where a frequency of $10 \mathrm{~Hz}$ was used. Unfortunately, the sampling rate of the original 13 bit Analogue to Digital Converter (ADC) was not high enough for this frequency, and a 12 bit ADC was used which did not allow differential inputs so that the accuracy of the measurement was reduced due to external electrical noise.

The solder paste sample length (see fig.1) for the data shown in fig. 11 is $1 \mathrm{~mm}$ compared to the $0.1 \mathrm{~mm}$ gap in fig.9, hence the resistance of the paste is increased. Although these measurements were taken $4.6 \mathrm{~s}$ after conduction began, so that the resistance is still dropping significantly, the underlying resistance drop over the duration of one triangular voltage pulse $(0 \mathrm{~V}-2 \mathrm{~V}-0 \mathrm{~V})$ is now negligible. The slight hystereses observed, with the resistance of the sample appearing to drop between the start and end of the measurement, is repeated throughout the experiment, and may be an indicator of bad synchronisation between the 4 voltage measurements required simultaneously in order to obtain one resistance measurement. There is also a slight possibility that the conduction observed is ionic conduction, and the hysteresis observed is related to electrochemical effects of the kind seen in cyclic voltammetry experiments. However the stability of the DC measurements over long time periods would tend to discount this possibility, as well as the fact that the majority of the solvents have been boiled off, though the temperature is below that required in order to melt the flux. 


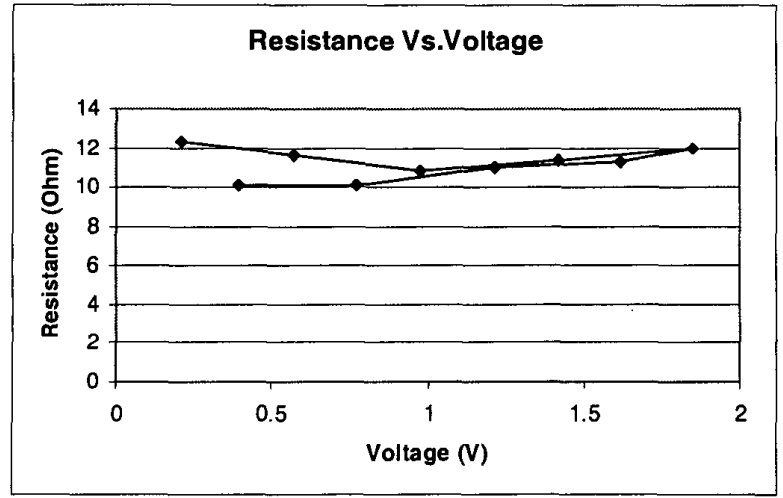

Figure 11. Measurements on solder paste at $10 \mathrm{~Hz}$ showing one triangular pulse $(0.05 \mathrm{~s})$

\section{Conclusions}

It has been shown how variation of solder paste resistance with temperature and time can be used to estimate contact spot sizes between solder paste particles. The activation energy of the process responsible for widening contact spots from the particle surfaces has been estimated and it is found that sintering is not the dominant mechanism. It is also unlikely that electrical contact between particles is prevented by a thin rosin layer coating the particles, as this would then be present until the rosin melts, well above $100^{\circ} \mathrm{C}$. The most likely candidate for the insulating layer on the particle surfaces is therefore an oxide. Furthermore, the steady erosion of these oxide layers may be caused by slow flux-oxide reactions, or by solder creep, or by a combination of the two.

Just before reflow, the resistance-voltage characteristics indicate that the contact spots are completely free of oxides, but that this is not the case at earlier stages of reflow. Fluxing reactions are seen to accelerate rapidly above $\sim 110^{\circ} \mathrm{C}$, while re-oxidation of the contact spots has not been observed in any of the experiments to date.

The size of the contact spots gives an indication of the extent to which oxide covers the particle surfaces before reflow begins. Reflow visualisation and CFD studies have shown that the oxide layer exerts a considerable restraining force on particles during reflow, and thus affects solder balling and incomplete wetting. [8] The evidence presented in section 3 shows that as reflow begins, oxide free areas $\sim 0.3 \mu \mathrm{m}^{2}$ are present between the particles, and future work will aim to correlate the extent of oxide free areas (hence efficacy of the flux) with soldering performance. One application of the proposed technique could be to monitor the degree of sintering in sintered solder joints. [9]

\section{Acknowledgments}

This work was conducted under UK EPSRC grants GR/L61767 and AF/990541, and with the help of an industrial consortium of partners including Multicore Solders Ltd., Celestica Europe Ltd., Mitel Semiconductor, DEK printing machines, Matra Dynamics and Intarsia Corp.

\section{References}

1. Mannan, S. H., et al, "Electrical method of monitoring percolation and abrasion of conducting spheres due to shear flow of a dense suspension in a narrow gap," Applied Physics Letters, Vol.75, no.6, (1999) pp. 871872.

2. Mannan, S. H., et al, "Flow Processes in Solder Paste during Stencil Printing for SMT Assembly," Jnl. of Materials Science: Materials in Electronics, Vol. 6, (1994) pp.34-42.

3. Holm, R., Electric contacts: theory and application,. 4th ed., Springer, (Berlin, 1967).

4. Ashby, M., "First Report on Sintering Diagrams," Acta Metallurgica, Vol.22, (1974) p273.

5. Peebles D., E., et al, "Kinetics of the isothermal spreading of tin on the air-passivated copper surface in the absence of a fluxing agent," Colloids and surfaces A, Vol. 144, (1998) pp.89-114.

6. Smith B., et al, "Understanding solder paste survivability and failure modes," Surface Mount Technology, (August 1988), pp.124-128.

7. Ohring, M., Materials science of thin films, Academic Press, (Boston; London: 1992.)

8. Mannan, S. H., et al, "Solder Paste Reflow Modelling for Flip Chip Assembly" To be published in proceedings of $3^{\text {rd }}$ Electronics Packaging Technology Conference, (December 2000, Sheraton Towers, Singapore).

9. Palmer M.A., et al "Forming solder joints by sintering eutectic tin-lead solder paste", Journal of Electronic Materials Vol. 28, (1999) pp.912-915. 